their health and wellbeing. The study can be used as a patient-centred approach for future planning and service provision.

\section{P-169 EFFECT OF A HOLISTIC APPROACH TO THE USE OF AQUATIC THERAPY FOR PALLIATIVE ADULT PATIENTS}

Charlotte Nicklin. Keech Hospice Care, Luton, UK

10.1136/bmjspcare-2019-HUKNC.191

\section{Background}

- Aquatic therapy is not standardly provided for palliative patients;

- A hydrotherapy pool provides a warm supportive environment for painful deconditioned bodies during or after medical treatment;

- Provides an opportunity to move, strengthen, relax and participate with significant effect on emotional well-being;

- Extends patient opportunities to therapies outside medical treatment, putting patients back in control.

Aims To evaluate the provision of aquatic therapy (therapistled hydrotherapy) for palliative patients by comparing objective measures and goals pre- and post- treatment

- Provide therapist-led aquatic therapy;

- Nurture and empower patients to become independent pool users.

Method Assess and work with palliative adult patients delivering aquatic therapy in 1:1 or group settings.

- Identify patient goals, physical baseline of ability including a range of standardised physical objective measures, patient perception of issues and well-being;

- 1:1 water-based assessment explores;

$\circ$ patient ability to exercise in water

○ water confidence

- trial personalised exercises and develop own written programme

- $<6$ further sessions of aquatic therapy 1:1, group or combination;

- Patient feedback questionnaire completed.

Results Data collected from all patients who completed a course of aquatic therapy, average duration two months.

- $100 \%$ improved physical function;

- $83 \%$ improved emotional well-being;

- $92 \%$ continued as independent pool users.

Conclusion Aquatic therapy can have a very positive, significant effect for patients affecting their physical function, willingness and motivation for exercise through their own perception of improvement, and emotional well-being. Relatively simple, short periods of interaction by a therapist can have meaningful long-term benefits for palliative adult patients for both physical outcomes and emotional well-being.

Innovation

- Currently no standardised use of aquatic therapy study demonstrates very positive outcomes for palliative patients including their perception, improved physical changes and function;

- Emphasis to empower patients with independent pool sessions in addition to attending a therapist-led session;
- Patients feel they are doing something to help themselves, which they find rewarding and motivating.

\section{P-170 MANAGING A CHANGE PROJECT - HOSPICE HYDROTHERAPY POOL}

Clare Burden. Keech Hospice Care, Luton, UK

10.1136/bmjspcare-2019-HUKNC.192

Background Hospice patient use of the hydrotherapy pool for aquatic therapy and memory work was minimal due to lack of information, unclear referral processes and no patient pathways for hydrotherapy.

Aims Increase hospice patient use of the pool by introducing clear referral pathways and having a structured Hydrotherapy pool team (Administrator and Lifeguards). For processes to be introduced that enabled individual members of the public with an appropriate need for hydrotherapy to access the pool and for external organisations to only use required spaces.

Methods Processes and access to the hydrotherapy pool by patients and their families, individual members of the public and external organisations were reviewed. Pool user expectations were managed, and changes communicated throughout. The structure and skill mix of the hydrotherapy pool team was reviewed and restructured. Care teams were involved in developing the pathways and referral process. Final versions of pathways were cascaded to teams.

Results Patient referrals for using the pool have increased and benefits are being recorded by the Aquatic Physiotherapist. It is expected that patient numbers will increase further. There is an increase in individual members of the public using the pool appropriately, through referrals from physiotherapists. External organisations are booking required number of spaces and confirming prior to use, thus allowing more patient and family spaces in the pool.

Conclusions Hospice patients and their families are a priority for using the hospice's hydrotherapy pool. Changes to the hydrotherapy processes and team have enabled appropriate access to the facility and increased use by patients.

\section{P-171 ANALYSING THE USE AND COST-EFFECTIVENESS OF DIAGNOSTIC ULTRASOUND IN A HOSPICE AND COMMUNITY SETTING}

${ }^{1,2}$ Nikki Reed, ${ }^{1}$ Kath Newton, 'Zameer Begum. 'Marie Curie West Midlands Hospice, Solihull, UK; ' University Hospitals Birmingham NHS Foundation Trust, Birmingham, UK

\subsection{6/bmispcare-2019-HUKNC.193}

Background Ultrasound is a diagnostic tool that can support clinical examination. Its use across the hospice and community palliative care setting can lead to significant cost savings with the avoidance of a hospital admission and/or the cost of hospital based interventional radiology service. Over the course of four years, 150 patients were clinically assessed with ultrasound by members of the hospice medical team (who had all received training in focussed abdominal ultrasound) and comprehensive details were recorded of their assessments.

Aim

- To ascertain if a hospice-based paracentesis service helped to reduce hospital admissions and/or interventional radiology input; 